\title{
Fairness based Improved Subcarrier Allocation Algorithms for OFDMA based Next Generation Networks
}

\author{
Rohit Sharma \\ E\&CE Department \\ NIT Hamirpur \\ Hamirpur, India
}

\author{
Manoranjan Rai Bharti \\ E\&CE Department \\ NIT Hamirpur \\ Hamirpur, India
}

\author{
Kamal Singh \\ E\&CE Department \\ NIT Hamirpur \\ Hamirpur, India
}

\begin{abstract}
Multiuser Orthogonal frequency division multiplexing (MUOFDM) is one of the promising techniques for future cellular and wireless networks for achieving high downlink capacities. The objective of the next generation cellular and wireless networks, which are based on (MU-OFDM) is to increase the network efficiency by maximizing the total throughput of the system and minimizing the overall transmit power for a given quality of service (QOS).
\end{abstract}

The sum capacity of MU-OFDM is maximized when each subchannel is assigned to the user with the best channel-tonoise ratio for that subchannel. Ever increasing demand for high throughput, low delay and low Outage probability can be met by using Adaptive Resource Allocation in OFDM Technology. In optimal resource allocation algorithms the subcarrier and power allocation should be done simultaneously but it is computationally complex. In order to reduce the complexity of resource allocation algorithms the subcarrier and power allocation is done separately and this is called sub-optimal subcarrier allocation and sub-optimal power allocation. Z. Shen et al. in [9] has given both suboptimal subcarrier allocation and sub-optimal power allocation algorithms. In this paper we have proposed two methods of subcarrier allocation based on subcarrier allocation method in [9], and compared them with subcarrier allocation algorithm of [9]. Comparison is made between subcarrier allocation algorithms only by considering equal power distribution among subcarriers instead of sub-optimal power allocation scheme given in [9]. The simulation results show the improvement in the sum capacity (total data rate achieved) with the proposed methods of subcarrier allocation over the subcarrier allocation in [9].

\section{General Terms}

Adaptive radio resource allocation, Rate adaptive radio resource allocation algorithm, Subcarrier allocation algorithm.

\section{Keywords}

Orthogonal frequency division multiplexing, sum capacity, fairness, subcarrier allocation.

\section{INTRODUCTION}

Orthogonal frequency division multiplexing (OFDM) is a promising technique for the next generation of wireless communication systems [1], [2]. OFDM is based on the concept of multicarrier transmission. In OFDM broadband channel is divided into $\mathrm{N}$ narrowband subchannels each with a bandwidth much smaller than the coherence bandwidth of the channel. The high rate data stream is then split into $N$ sub- streams of lower rate data which are modulated into $N$ OFDM symbols and transmitted simultaneously on $N$ orthogonal subcarriers. Besides the improved immunity to fast fading [3] brought by the multicarrier property of OFDM systems, multiple access is also possible, because the subchannels are orthogonal to each other.

The network efficiency in OFDMA systems can be improved by allocating radio resources intelligently. There are two classes of radio resource allocation schemes: 1) fixed resource allocation [4]; and 2) Adaptive or dynamic resource allocation [5]-[8]. Fixed resource allocation schemes, such as time division multiple access (TDMA) and frequency division multiple access (FDMA), assign an independent dimension, e.g., time slot or subchannel, to each user. A fixed resource allocation scheme is not optimal, since the scheme is fixed regardless of the current channel conditions. On the other hand, dynamic resource allocation scheme allocates a dimension adaptively to the users based on their channel gains. Due to the time-varying nature of the wireless channel, dynamic resource allocation makes full use of multiuser diversity to achieve higher performance.

There are two classes of optimization techniques for adaptive resource allocation, proposed in the literature, namely: 1) margin adaptive (MA) [5]; and 2) rate adaptive (RA) [6], [7]. The MA objective is to achieve the minimum overall transmit power given the constraints on the users' data rates or bit error rates (BER). The RA objective is to maximize each user's error free capacity with a total transmit power constraint [10].

Adaptive Resource Allocation algorithm in Multiuser OFDM systems with Proportional Rate Constraints has been proposed in [9]. In [9] subcarrier and power allocation has been done separately. In our paper we have proposed two methods of subcarrier allocation based on [9] considering equal power distribution among subcarriers instead of sub optimal power allocation given in [9]. Both the proposed methods result in improvement of sum data rate achieved over subcarrier allocation method in [9].

The paper is organized as follows; Section 2 introduces the multiuser OFDM system model and presents the optimization objective function. In Section3, the two proposed methods of subcarrier allocation are given. In section 4, simulation results are presented and in section 5, conclusions are drawn. 


\section{SYSTEM MODEL}

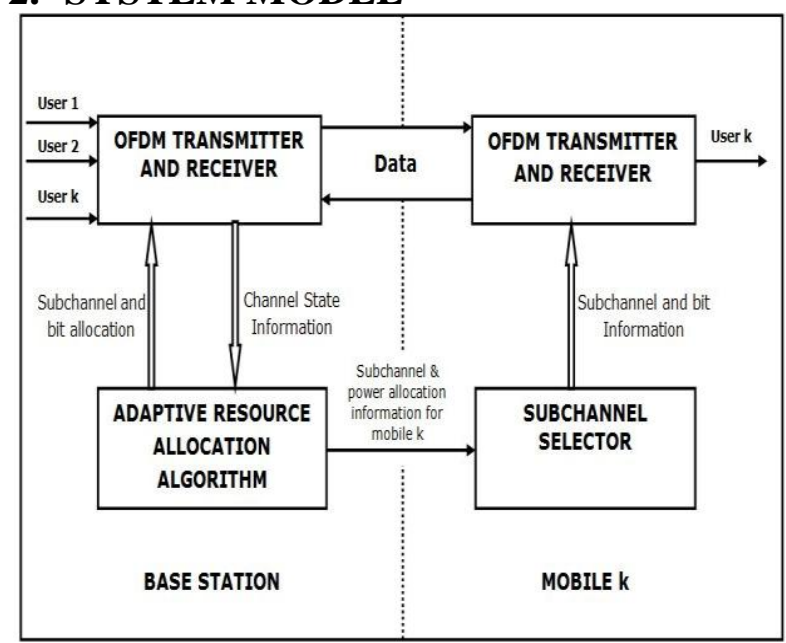

Fig 1: Multiuser OFDM Block Diagram

At the base station, the OFDM receiver collects all the channel state information from the users through a feedback system and passes this information to the adaptive resource allocation block. The resource allocation done is then passed on to the OFDM transmitter. The resource allocation scheme is updated as fast as the channel information is collected. In this paper, perfect instantaneous channel information is assumed to be available at the base station.

In this paper, we assume a total of $\mathrm{K}$ users in the system sharing $\mathrm{N}$ subchannels, with total transmit power constraint $P_{\text {total }}$. Our objective is to optimize the subchannel allocation in order to achieve the highest sum error free capacity under the total power constraint. Our objective function is equally weighted sum capacity with proportional fairness also being considered in the system. The advantage of considering proportional fairness in the system is that the capacity ratio among users can be explicitly controlled, and this will ensure that each user is able to meet his target data rate.

Mathematically, the optimization problem can be described as:

$$
\begin{aligned}
& \max _{p_{k, n}, \rho_{k, n}} \sum_{k=1}^{K} \sum_{N=1}^{N} \frac{\rho_{k, n}}{N} \log _{2}\left(1+\frac{p_{k, n} h_{k, n}^{2}}{N_{o} \frac{B}{N}}\right) \\
& \text { subject to } \sum_{k=1}^{K} \sum_{n=1}^{N} p_{k, n} \leq P_{\text {total }} \\
& p_{k, n} \geq 0 \quad \text { for all } \mathrm{k}, \mathrm{n} \\
& \rho_{k, n}=\{0,1\} \text { for all } \mathrm{k}, \mathrm{n} \\
& \sum_{k=1}^{K} \rho_{k, n}=1 \text { for all } \mathrm{n}
\end{aligned}
$$$$
R_{1}: R_{2}: \ldots: R_{k}=\gamma_{1}: \gamma_{2}: \ldots: \gamma_{K}
$$

where $\mathrm{K}$ is the total number of users, $\mathrm{N}$ is the total number of subcarriers, $N_{0}$ is the power spectral density of AWGN channel, B and $P_{\text {total }}$ are the total available bandwidth and power, respectively, ${ }_{k, n}$ is the power allocated for user $\mathrm{k}$ on the subcarriers $\mathrm{n}, h_{k, n}$ is the channel gain for user $\mathrm{k}$ on subcarriers $\mathrm{n}$, and $\rho_{k, n}$ can only be either 1 or 0 , indicating whether subcarriers $\mathrm{n}$ is used by user $\mathrm{k}$ or not. The fourth constraint shows that each subcarrier can only be used by one user.

The capacity for user $k$ is denoted as:

$$
R_{k}=\sum_{n=1}^{N} \frac{\rho_{k, n}}{N} \log _{2}\left(1+\frac{p_{k, n} h_{k, n}^{2}}{N_{0} \frac{B}{N}}\right)
$$

Finally $\left\{\gamma_{i}\right\}_{i=1}^{K}$ is a set of predetermined values that are used to ensure proportional fairness among users.

\section{MODIFIED SUBCARRIER \\ ALGORITHMS} SUBOPTIMAL ALLOCATION

Ideally for optimal solution of (1) subchannel and power should be allocated jointly. However it poses a prohibitive computational burden at the base station for obtaining the optimal solution. Furthermore, the base station has to rapidly compute the optimal subchannel and power allocation as the wireless channel changes. Hence, for cost-effective and delaysensitive implementations, low-complexity suboptimal algorithms are preferred. In this section, we are proposing two suboptimal subchannel allocation algorithms. Sections 3.1 presents proposed method-1 of subchannel allocation scheme and Section 3.2 presents proposed method-2 of subchannel allocation scheme. In both the schemes equal power distribution among the subcarriers has been considered.

\subsection{Proposed Method-1}

In this sub-section we are presenting a suboptimal subchannel allocation algorithm which is our proposed method-1. Let $H_{k, n}=\left(h_{k, n}^{2} / N_{0}(B / N)\right)$ as the channel-to-noise ratio for user $\mathrm{k}$ in subchannel $\mathrm{n}$ and $\Omega_{k}$ is the set of subchannels assigned to user $\mathrm{k}$. The algorithm is described as follows:

1. Initialization

$$
\begin{aligned}
& \text { Set } R_{k}=0, \Omega_{k}=\phi \text { for } \mathrm{k}=1,2, \ldots ., \mathrm{K} \text { and } \\
& \mathrm{A}=\{1,2, \ldots \ldots, \mathrm{N}\} ; K^{\prime}=\{1,2, \ldots, K\} .
\end{aligned}
$$

2. While $K^{\prime} \neq \phi$

(a) Find k, n such that $\left|H_{k, n}\right| \geq\left|H_{l, j}\right|$ for all $\mathrm{j} \in \mathrm{A}$ and $l \in K^{\prime}$.

(b) For the found $\mathrm{k}$ and $\mathrm{n}$, let $\Omega_{k}=\Omega_{k} \cup\{n\}$, $\mathrm{A}=\mathrm{A}-\{\mathrm{n}\}, K^{\prime}=K^{\prime}-\{k\}$ and update $\boldsymbol{R}_{k}$ according to (2). 
3. While $A \neq \phi$

(a) Find $\mathrm{k}$ satisfying $R_{k} / \gamma_{k} \leq R_{i} / \gamma_{i}$ for all i, $1 \leq i \leq K$

(b) For found k, find n satisfying $\left|H_{k, n}\right| \geq\left|H_{k, j}\right|$ for all $\mathrm{j} \in \mathrm{A}$;

(c) For the found $\mathrm{k}$ and $\mathrm{n}$, let $\Omega_{k}=\Omega_{k} \cup\{n\}$, $\mathrm{A}=\mathrm{A}-\{\mathrm{n}\}$ and update $R_{k}$ according to (2).

This algorithm is based on [9], but we have proposed some modifications as described above. The proposed modifications have been found to show improvement in the sum data rate achieved over the method in [9]. The subcarrier allocation algorithm in [9], the second step (first loop) allocates one subcarrier to each user by picking one user $(\mathrm{k})$ randomly and allocating best subcarrier (n) to that user. The gain of that subcarrier could be more for another user. Considering this fact in our proposed method-1, in second step, we find best user-subcarrier combination i.e. best $\mathrm{k}-\mathrm{n}$ combination. Based on this combination, we allocated one subcarrier to each user. In next step the remaining subcarriers are allocated in the same way as they are allocated in [9] to introduce fairness. In this suboptimal subchannel allocation algorithm, equal power distribution is assumed across all subcarriers.

\subsection{Proposed Method-2}

In this sub-section, we are proposing one more suboptimal subchannel allocation algorithm (proposed method-2 algorithm). Let $H_{k, n}=\left(h_{k, n}^{2} / N_{0}(B / N)\right)$ as the channel-tonoise ratio for user $\mathrm{k}$ in subchannel $\mathrm{n}$ and $\Omega_{k}$ is the set of subchannels assigned to user $\mathrm{k}$. This algorithm is as follows:

First we calculate a factor $\mathrm{N}^{*}$

$$
\begin{aligned}
& N_{k}=\left[N\left(\frac{\gamma_{k}}{\sum_{i=1}^{K} \gamma_{i}}\right)-1\right] \\
& N^{*}=\sum_{i=1}^{K} \operatorname{int}\left(N_{i}\right)
\end{aligned}
$$$$
\mathrm{k}=1,2, \ldots, \mathrm{K}
$$

Here the function int $(\cdot)$ rounds the value of $\mathrm{N}_{\mathrm{i}}$ to the nearest integers less than or equal to $\mathrm{N}_{\mathrm{i}} . \mathrm{N}_{\mathrm{k}}$ is the number which is one less than the total number of subcarriers required by user $\mathrm{k}$ proportional to its data rate requirement.

1. Initialization

$$
\begin{aligned}
& \text { Set } R_{k}=0, \Omega_{k}=\phi, \text { for } \mathrm{k}=1,2, \ldots . ., \mathrm{K} \text { and } \\
& \mathrm{A}=\{1,2, \ldots ., \mathrm{N}\} ; K^{\prime}=\{1,2, \ldots, K\} .
\end{aligned}
$$

2. While $\|A\|>N^{*}$

(a) Find $\mathrm{k}$, n such that $\left|H_{k, n}\right| \geq\left|H_{l, j}\right|$ for all $\mathrm{j} \in \mathrm{A}$ and $l \in K^{\prime}$. (b) For the found $\mathrm{k}$ and $\mathrm{n}$, let $\Omega_{k}=\Omega_{k} \cup\{n\}$, $\mathrm{A}=\mathrm{A}-\{\mathrm{n}\}$, and update $\boldsymbol{R}_{\boldsymbol{k}}$ according to (2).

3. While $K^{\prime} \neq \phi$

(a) Find k that satisfies $R_{k} \leq R_{i}$ for all i, $i \in K^{\prime}$;

(b) For the found k, find n satisfying $\left|H_{k, n}\right| \geq\left|H_{k, j}\right|$ for all $\mathrm{j} \in \mathrm{A}$;

(c) For the found $\mathrm{k}$ and $\mathrm{n}$, let $\Omega_{k}=\Omega_{k} \cup\{n\}$, $\mathrm{A}=\mathrm{A}-\{\mathrm{n}\}, K^{\prime}=K^{\prime}-\{k\}$ and update $\boldsymbol{R}_{k}$ according to (2).

4. While $A \neq \phi$

(a) Find $\mathrm{k}$ satisfying $R_{k} / \gamma_{k} \leq R_{i} / \gamma_{i}$ for all i, $1 \leq i \leq K$

(b) For found k, find n satisfying $\left|H_{k, n}\right| \geq\left|H_{k, j}\right|$ for all $\mathrm{j} \in \mathrm{A}$;

(c) For the found $\mathrm{k}$ and $\mathrm{n}$, let $\Omega_{k}=\Omega_{k} \cup\{n\}$, $\mathrm{A}=\mathrm{A}-\{\mathrm{n}\}$ and update $R_{k}$ according to (2).

Here $\|A\|$ is the cardinality of set $\mathrm{A}$.

In this proposed method-2, the second step (first loop) allocates subcarriers to the users based on best user-subcarrier combination i.e. best $\mathrm{k}-\mathrm{n}$ combination. The number of subcarriers allocated in this step is determined by the factor $\mathrm{N}^{*}$. In the third step (second loop), we allocate one subcarrier to each user such that the user, who has minimum achieved data rate in the second step, is allocated channel first. In the fourth step (third loop) of proposed method-2, the remaining subcarriers are allocated according to third step (second loop) of subcarriers allocation algorithm given in [9]

This algorithm is also based on [9] but the proposed modifications have been found to show improvement in the sum data rate achieved over subcarrier allocation method in [9]. In this suboptimal subchannel allocation algorithm, equal power distribution is assumed across all subcarriers.

\section{SIMULATION RESULTS}

We have done simulation to compare the performance of our proposed subcarrier allocation methods and subcarrier allocation algorithm in [9]. In all simulations presented in this section, the wireless channel is modelled as a frequencyselective channel. Simulation has been done in Matlab and following parameters have been considered for simulation purpose:

Table1: Simulation Parameters

\begin{tabular}{|l|l|}
\hline $\begin{array}{l}\text { Total Power Available at the Base } \\
\text { Station }\end{array}$ & $1 \mathrm{~W}$ \\
\hline AWGN power spectral density & $-70 \mathrm{dBW} / \mathrm{Hz}$ \\
\hline Overall Bandwidth & $1 \mathrm{MHz}$ \\
\hline Number of OFDMA subchannels & 64 \\
\hline
\end{tabular}


The simulation results are shown in figure 2, figure 3 and figure 4 as follows:

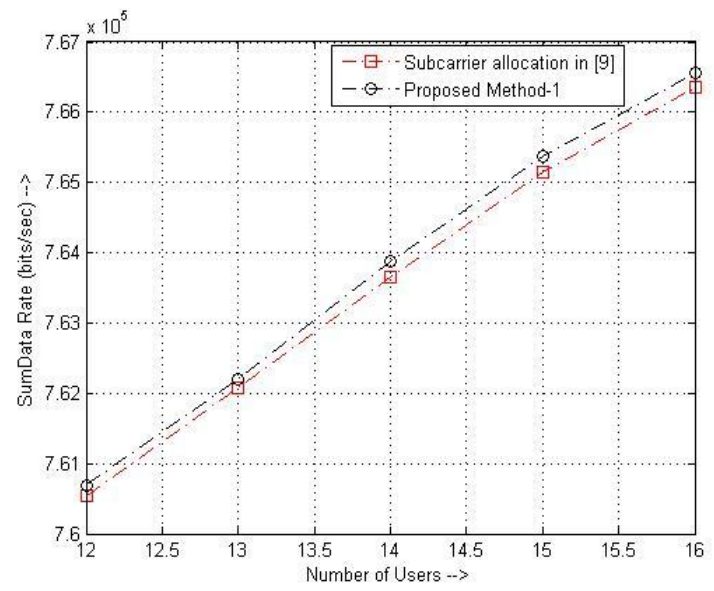

Fig 2: Comparison of sum data rate v/s number of users for subcarrier allocation method in [9] and proposed subcarrier allocation method-1

Figure 2 shows the comparison of subcarrier allocation algorithm in [9] and proposed method-1 of subcarrier allocation on the basis of sum data rate. With equal power distribution among subcarriers being considered, the sum data rate achieved by proposed method- 1 is more than the subcarrier allocation method in [9]. Whereas the second step (first loop) of [9] allocates one subcarrier to each user picking users one by one, our proposed method-1 allocates one subcarrier to each user by finding best user-subcarrier combination. This modification gives us the improvement in sum data rate achieved which is verified by simulation results.

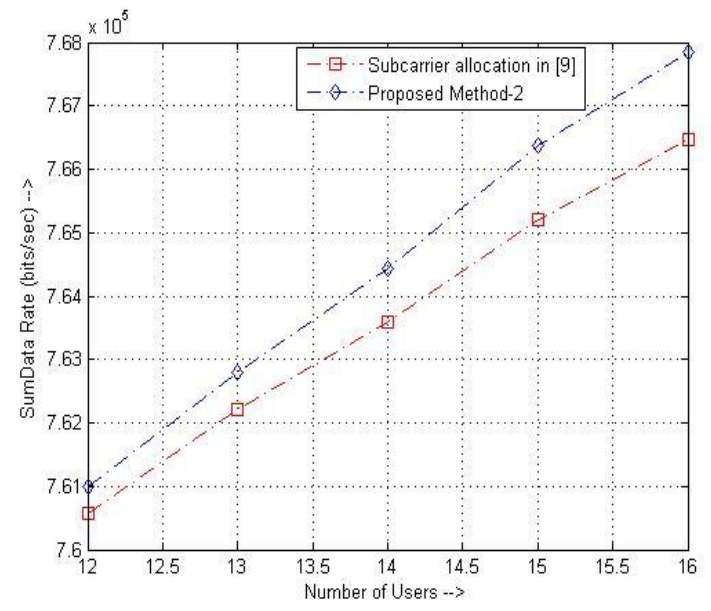

Fig 3: Comparison of sum data rate v/s number of users for subcarrier allocation method in [9] and proposed subcarrier allocation method-2

Figure 3 shows the comparison of subcarrier allocation algorithm in [9] and proposed method-2 of subcarrier allocation on the basis of sum data rate. With equal power distribution among subcarriers being considered, the sum data rate achieved by proposed method- 2 is more than the subcarrier allocation method in [9]. In the proposed method-2, second step (first loop), few subcarriers are allocated to user(s) based on best user-subcarrier combination among all the available subcarriers. The third step (second loop) of proposed method- 2 allocates one subcarrier to each user such that the user with minimum achieved data rate in the second step is allocated channel first. In the fourth step (third loop) of proposed method-2, the remaining subcarriers are allocated according to third step (second loop) of subcarriers allocation algorithm given in [9]. These modifications give us the improvement in sum data rate achieved which is verified by simulation results.

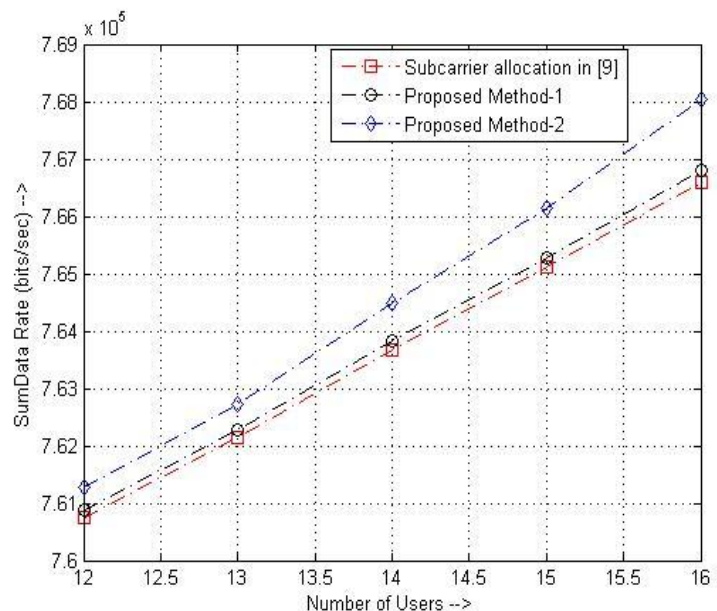

Fig 4: Comparison of sum data rate v/s number of users for subcarrier allocation method in [9], proposed subcarrier allocation method-1 and method-2

Figure 4 shows the comparison of proposed method-1, proposed method-2 and subcarrier allocation method in [9]. As can be seen, the proposed method-2 provides more improvement in sum data rate achieved over the proposed method-1. Simulation results also show that both the proposed methods give improvement in the achievable sum data rate when compared with subcarrier allocation method of [9].

\section{CONCLUSION}

This paper proposed two suboptimal subchannel allocation methods for the downlink of a multi-user OFDM system. Though the methods are based on [9], however, simulation results indicate that the proposed methods provide improvement for sum data rate achieved when compared with subcarrier allocation method in [9]. In all the simulations we have considered equal power distribution among subcarriers. The proposed method- 2 provides more improvement in the sum data rate than the proposed method-1 as shown in figure 4; but the proposed method-1 provides more fairness than the proposed method-2. In the proposed method-1, proposed method-2 and subcarrier allocation method in [9], the sum data rate increases with the number of users which can be explained by the multiuser diversity. This means as the number of users in the system increases it lowers the probability that a given subchannel is in a deep fade for all users.

\section{REFERENCES}

[1] H. Sampath, S. Talwar, J. Tellado, V. Erceg, and A. Paulraj, "A fourth generation MIMO-OFDM broadband wireless system: Design, performance, and field trial results," IEEE Commun. Mag., vol. 40, no. 9, pp. 143149, Sep. 2002.

[2] T. S. Rappaport, A. Annamalai, R. M. Buehrer, and W. H. Tranter, "Wireless communications: Past events and a future perspective," IEEE Commun. Mag., vol. 40, no. 5, pp. 148-161, May 2002. 
[3] J. A. C. Bingham, "Multicarrier modulation for data transmission: An idea whose time has come," IEEE Commun. Mag., vol. 28, no. 5, pp. 5-14, May 1990.

[4] E. Lawrey, "Multiuser OFDM," in Proc. Int. Symp. Signal Processing and Its Applications, Brisbane, Australia, 1999, pp. 761-764.

[5] C. Y. Wong, R. S. Cheng, K. B. Letaief, and R. D. Murch, "Multicarrier OFDM with adaptive subcarrier, bit, and power allocation," IEEE J. Sel. Areas Commun., vol. 17, no. 10, pp. 1747-1758, Oct. 1999.

[6] J. Jang and K. B. Lee, "Transmit power adaptation for multiuser OFDM systems," IEEE J. Sel. Areas Commun., vol. 21, no. 2, pp. 171-178, Feb. 2003.

[7] W. Rhee and J. M. Cioffi, "Increasing in capacity of multiuser OFDM system using dynamic subchannel allocation," in Proc. IEEE Int. Vehicular Tech. Conf., Tokyo, Japan, May 2000, vol. 2, pp. 1085-1089.
[8] I. Kim, H. L. Lee, B. Kim, and Y. H. Lee, "On the use of linear programming for dynamic subchannel and bit allocation in multiuser OFDM," in Proc. IEEE Global Communications Conf., San Antonio, TX, 2001, vol. 6, pp. 3648-3652.

[9] Zukang Shen, Jeffrey G. Andrews, Brian L. Evans, "Adaptive Resource Allocation in Multiuser OFDM Systems with Proportional Rate Constraints,' IEEE transactions on wireless communications, vol. 4, no. 6 , pp. 2726-2737, November 2005.

[10] Rohit Sharma and Manoranjan Rai Bharti, "Adaptive Resource Allocation with Proportional Rate Constraints for OFDMA Based Next Generation Networks," IJCA Special Issue on Communication and Networks comnetcn(1):40-43, December 2011. Published by Foundation of Computer Science, New York, USA. 